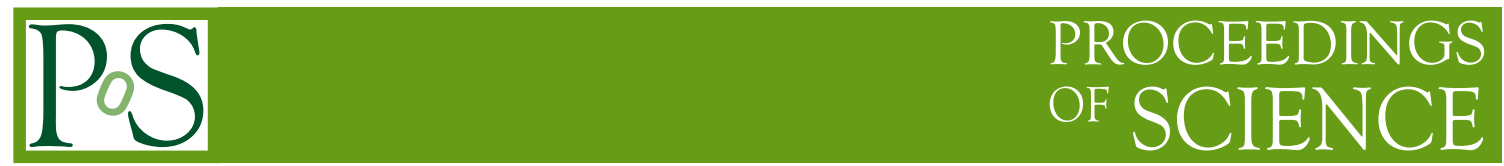

\title{
Computation of the string tension in three dimensions using large $\mathbf{N}$ reduction
}

\section{Joseph Kiskis*}

Department of Physics, University of California, Davis, CA 95616, USA

E-mail: jekiskiseucdavis.edu

\section{Rajamani Narayanan}

Department of Physics, Florida International University, Miami, FL 33199, USA

E-mail: rajamani.narayananefiu.edu

We numerically compute the string tension in the large $\mathrm{N}$ limit of three dimensional Yang-Mills theory using Wilson loops. Space-time Wilson loops are formed using smeared space-like links and unsmeared time-like links. We use partial reduction and both unfolded and folded Wilson loops in the analysis.

The XXVI International Symposium on Lattice Field Theory

July 14 - 19, 2008

Williamsburg, Virginia, USA

\footnotetext{
* Speaker.
} 


\section{Introduction}

The method of large $N$ continuum reduction [1], for for $S U(N)$ gauge theory allows for the calculation of the infinite volume, infinite $N$ limit of certain physical quantities using volumes reduced to a small physical size. Numerical estimates [1] 2- of the physical critical size above which continuum reduction holds indicate that this method can be used to produce practical results. The chiral condensate [3] and the pion decay constant[4] were calculated in the large $N$ limit in four dimensions using continuum reduction. In this paper, we show that the method can be extended beyond bulk quantities and that it also produces reliable results for quantities with spacetime dependence such as the heavy quark potential, from which the string tension can be extracted. Invoking large $N$ continuum reduction, we included Wilson loops larger than the size of the lattice. The results validate the method of continuum reduction for calculating quantities based on the space-time dependence Wilson loops.

A precise calculation of the string tension in three dimensional $S U(N)$ gauge theories has been performed with $N$ up to 8 on large lattices [5]. We present a complementary calculation with $N=47$ on $5^{3}$ lattices using continuum reduction. The calculation of Ref. [5] used correlation functions of smeared Polyakov loops to extract the string tension. After extrapolating to $N=\infty$ and to the continuum, the result was

$$
\frac{\sqrt{\sigma}}{g^{2} N}=0.1975 \pm 0.0002-0.0005
$$

where $g$ is the gauge coupling. This has to be compared with the analytical calculation in [7], namely, $\frac{1}{\sqrt{8 \pi}} \approx 0.1995$. Although the two results are not in perfect agreement, the main observation is that the approximations used in the analytical calculation are very well motivated.

Our use of continuum reduction to directly compute the $N=\infty$ limit of the string tension by working at large enough $N$ so that the finite $N$ corrections are smaller than the numerical errors gives [6]

$$
\frac{\sqrt{\sigma}}{g^{2} N}=0.1964 \pm 0.0009
$$

This result and that of (1.1) are consistent at the level of their one sigma errors. This level of agreement is, in turn, consistent with neither the large $N$ extrapolation of Ref. [5] nor the volume reduction of the present calculation having unexpected errors. While both of the numerical results lie below the analytical estimate, the discrepancy is relatively small. Thus the numerical evidence that the analytical result is an excellent first approximation that captures much of the physics remains strong.

The paper is organized as follows. We explain how we use smeared Wilson loops to compute the string tension in Section 2. The lattice results for the string tension along with the continuum extrapolation are also presented in this section. An intermediate step in our calculation is the dimensionless ground state string energy $m(k)$. In Section 3, we show results for $m(k)$ at one fixed lattice coupling to illustrate its behavior as a function of $k$ and how it is used to extract the string tension. We also show that $m(k)$ is unaffected by the smearing parameter. We illustrate the extraction of $m(k)$ at one fixed coupling in Section 4 . Here we show how the smearing parameter affects the overlap with the ground state. The main result in this paper is obtained using $N=47$. 
We show that the finite $N$ and finite volume corrections are small at this value of $N$ in Section 5. We explain why this method is preferred over the Creutz ratio in Section 6.

\section{String tension using Wilson loops and continuum reduction}

Consider $S U(N)$ Yang-Mills theory on a periodic lattice with the standard Wilson gauge action. The method of [5] is to measure the string tension using correlations of Polyakov loops with separation $t$ that wind around a space direction. Continuum reduction [1, 2] implies that the large $N$ Yang-Mills theory in a continuum box of size $l^{3}$ is independent of $l$ as long as $l>l_{c}=1 / T_{c}$ with $T_{c}$ being the deconfining temperature. One should be able to compute expectation values of Wilson loops of arbitrary size on an $l^{3}$ continuum box using folded Wilson loops and extract the string tension. To implement this approach to the three-dimensional Yang-Mills theory string tension, we use the following procedure:

- We fix the lattice size to $L^{3}$. We use $L=5$ for the most part and only use $L=4$ to verify reduction.

- We fix $N$ so that finite $N$ corrections are small. We set $N=47$ and show using one instance that finite $N$ corrections are small at $N=47$.

- We pick an appropriate range of lattice coupling $b=\frac{1}{g^{2} N}$.

- $b$ cannot be too small since we have to be away from the bulk transition on the lattice associated with the development of gap in the eigenvalue distribution of the plaquette operator [8]. Therefore, we pick $b \geq 0.6$.

- $b$ cannot be too big since we have to be below the deconfining transition for $L=5$. Therefore, we pick $b \leq 0.8$ [9].

- We use smeared space-like links and unsmeared time-like links.

- We use the tadpole improved coupling $b_{I}=b e(b)$ to set the scale and consider $K \times T$ Wilson loops $W(K, T)$ with $1.5<\frac{K}{b_{I}}, \frac{T}{b_{I}}<12.5$. This amounts to expectation values of Wilson loops that range from 0.82 to $2 \cdot 10^{-4}$.

- Keeping $K$ fixed, we fit

$$
\ln W(k, t)=-a-m(k) t
$$

where $k=\frac{K}{b_{I}}$ and $t=\frac{T}{b_{I}}$ are the dimensionless extent in the space and time direction respectively. $m(k)$ is the dimensionless ground state energy. This fit assumes that there is a perfect overlap with the ground state. Note that $a$ should be zero since $W(k, 0)=1$. Any small deviation from zero seen in the fit is due to the contribution from excited states.

- Finally, $m(k)$ is fit to $\sigma b_{I}^{2} k+c_{0} b_{I}+\frac{c_{1}}{k}$. The combination $\sqrt{\sigma} b_{I}$ is plotted as a function of $b_{I}^{-2}$. We expect lattice spacing effects to lead off as $b_{I}^{-2}$ in Yang-Mills theories and this is indeed the case in Fig. 1. The continuum limit extracted from this figure was quoted in Eqn.(1.2). 


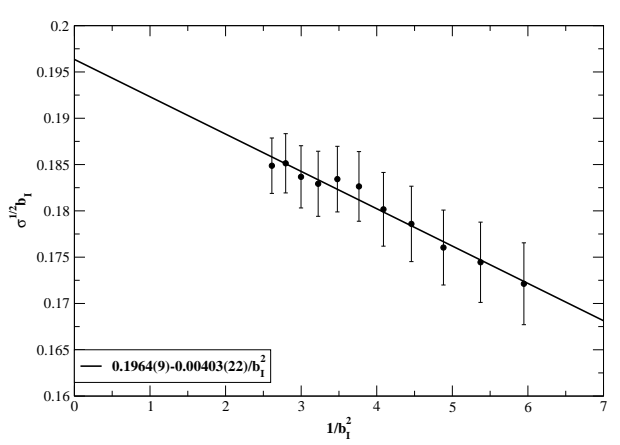

Figure 1: The string tension is plotted as a function of the lattice spacing $b_{I}^{-1}$. The fit is an extrapolation to the continuum.

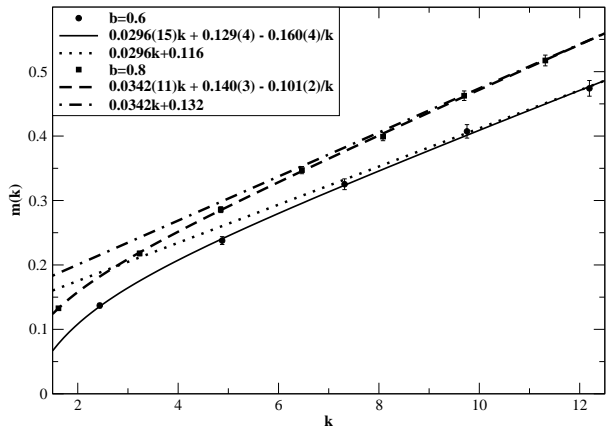

Figure 2: The ground state energy $m(k)$ as a function of $k$ for the coarse and fine lattice spacings considered here.

The use of smeared links improves the measurement of Wilson loops. They enhance the overlap of the space-like sides of the Wilson loops with the ground state. This increases the signal relative to the fluctuations and simplifies the $t$ behavior of the loops [10]. One step in the iteration takes one from a set $U_{k}^{(i)}\left(x_{1}, x_{2}, t\right)$ to a set $U_{k}^{(i+1)}\left(x_{1}, x_{2}, t\right)$. Before reunitarization, the weight of $U_{k}^{(i)}\left(x_{1}, x_{2}, t\right)$ is $(1-f)$ while that of each staple is $f / 2$. The time-like links, $U_{3}\left(x_{1}, x_{2}, t\right)$, are not smeared, and the smearing only involves space-like staples. There are two parameters, namely, the smearing factor $f$ and the number of smearing steps $n$. Only the product $\tau=f n$ matters, and $f$ plays the role of a discrete smearing step. For a given $\tau$, the overlap of the smeared loop with the ground state does not depend on $f$ as long as it is small. But the overlap of the smeared loop with the ground state does depend upon $\tau$. We set the value of the smearing parameter to $\tau=2.5$ by choosing $f=0.1$ and $n=25$. To study the effect of varying $\tau$, we also consider $\tau=1.25$ ( $f=0.05$ and $n=25$ ) at one coupling.

\section{Extraction of string tension}

$S U(N)$ gauge fields were generated on a $5^{3}$ periodic lattice using the standard Wilson action. One gauge field update of the whole lattice [2] is one Cabibbo-Marinari heat-bath update of the whole lattice followed by one $S U(N)$ over-relaxation update of the whole lattice. A total of 1500 such updates were used to achieve thermalization. Measurements were separated by 10 such updates and all estimates are from a total of 832 such measurements. Errors in all quantities at a fixed $b$ and $N$ were obtained by jackknife with single elimination.

The ground state energy $m(k)$ obtained as a function of $k=\frac{K}{b_{I}}$ is fit to

$$
m(k)=\sigma b_{I}^{2} k+c_{0} b_{I}+\frac{c_{1}}{k}
$$

We expect $\sigma b_{I}^{2}$ to approach a finite value in the continuum limit $\left(b_{I} \rightarrow \infty\right)$. The three parameter fit of $m(k)$ as a function of $k$ is shown in Fig. 2.

\section{Extraction of $m(k)$}

The dimensionless ground state energy $m(k)$ is extracted at a fixed $k$ by fitting $\ln W(k, t)$ to 


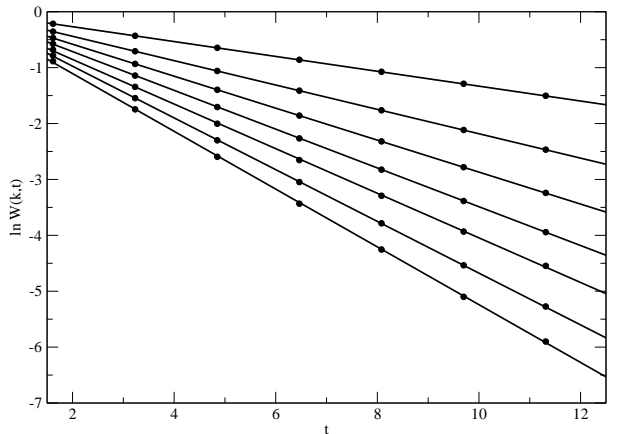

Figure 3: Plot of $\ln W(k, t)$ as a function of $t$ for seven different values of $k$ at $b=0.8$ with $\tau=2.5$.

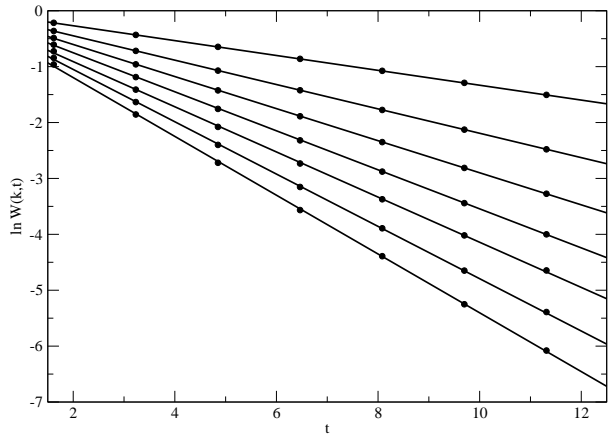

Figure 4: Plot of $\ln W(k, t)$ as a function of $t$ for seven different values of $k$ at $b=0.8$ with $\tau=1.25$.

$-a-m(k) t$ as discussed in Sec. 2. While $m(k)$ should be independent of the smearing parameter $\tau=f n$, the value of $a$ is expected to depend on $\tau$.

We will use $b=0.8$ as the coupling to illustrate the extraction of $m(k)$. Figure 3 and Fig. $\$$ show the performance of the fit for two different values of $\tau$, namely, 2.5 and 1.25 respectively. The solid circles show the data points without errors. The solid lines show the fit of the data. Seven values of $t$ were used to fit the data at one $k$, and data at seven different values of $k$ were fitted. This amounted to all Wilson loops from $1 \times 1$ to $7 \times 7$ on the $5^{3}$ lattice. The fit parameters are shown in Table 11 and Table 2 . Only the average values of the fit parameters are listed.

Investigation of Table 1 and Table 2 shows that $m(k)$ does not depend on $\tau$. There is a small difference in the two values of $m(k)$ at a fixed $k$ for the two different values of $\tau$ if $k$ is large. Additional analysis shows that this difference is within errors. Furthermore, the fitted values of $\sigma b_{I}^{2}$ for the two different values of $\tau$ are the same within errors.

$\begin{array}{cccccccc}k & 1.62 & 3.23 & 4.85 & 6.47 & 8.08 & 9.70 & 11.31 \\ a & 0.001 & 0.003 & 0.009 & 0.019 & 0.055 & 0.047 & 0.071 \\ m(k) & 0.133 & 0.218 & 0.286 & 0.347 & 0.399 & 0.464 & 0.517\end{array}$

Table 1: Fit parameters corresponding to the fit $\ln W(k, t)=-a-m(k) t$ for seven different values of $k$ at $b=0.8$ with $\tau=2.5$.

$\begin{array}{cccccccc}k & 1.62 & 3.23 & 4.85 & 6.47 & 8.08 & 9.70 & 11.31 \\ a & 0.002 & 0.012 & 0.029 & 0.054 & 0.102 & 0.114 & 0.144 \\ m(k) & 0.133 & 0.218 & 0.287 & 0.349 & 0.404 & 0.468 & 0.526\end{array}$

Table 2: Fit parameters corresponding to the fit $\ln W(k, t)=-a-m(k) t$ for seven different values of $k$ at $b=0.8$ with $\tau=1.25$.

The values of $a$ in Table 1 and Table 1 do show a variation with $\tau$ and $k$. Since a smaller value of $\tau$ implies less smearing, the overlap with the ground state is less for smaller $\tau$, and this results in a larger value of $a$ at smaller $\tau$. The value of $a$ is very close to zero for small $k$ indicating excellent overlap with the ground state for the chosen value of $\tau$. As $k$ increases, the length of the loop increases and the perimeter divergence has a stronger effect. This results in a larger value of $a$ as $k$ increases at a fixed $\tau$. 


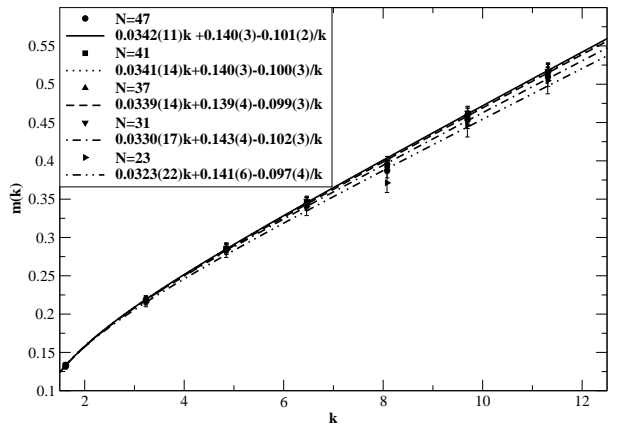

Figure 5: The ground state energy $m(k)$ as a function of $k$ for five different values of $N$

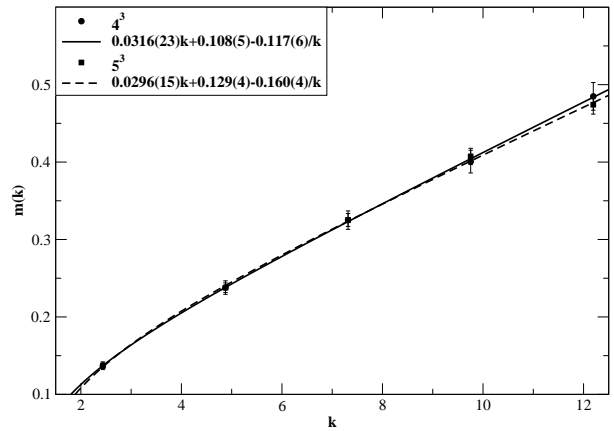

Figure 6: The ground state energy $m(k)$ as a function of $k$ on two different lattices at $b=0.8$.

\section{Finite $N$ effects}

Two issues need to be addressed with the analysis performed so far. We have fixed our value of $N$ assuming finite $N$ effects are small. If $N$ is not large enough, finite $N$ effects need to be addressed. In addition, we also have to address finite volume effects since continuum reduction is valid only in the $N \rightarrow \infty$ limit.

We expect $m(k)$ to have a fixed limit as $N \rightarrow \infty$ at a fixed $k, L, b$ and $\tau$. Indeed, this is the case as shown in Fig. 5 where the results for $m(k)$ as a function of $k$ are shown for $b=0.8$ with $\tau=2.5$ on $5^{3}$ lattice. All three fit parameters are consistent within errors all the way from $N=23$ to $N=47$. The only glitch one sees is at $k \approx 8$. This corresponds to $K=k b_{I}=5$, which is the linear extent of the lattice. One can argue that there are larger finite $N$ effects at strong coupling for $K=L$. Since the fit of $m(k)$ involves several values of $k$, the larger effect at this particular value of $k$ is diminished in the extraction of $\sigma b_{I}^{2}$.

Since finite $N$ effects can be ignored at $N=47$, we also expect there to be no appreciable finite volume effects at this value of $N$. This point is illustrated in Fig. 6 where the result for $m(k)$ is plotted at $b=0.6$ and $\tau=2.5$ on $4^{3}$ and $5^{3}$ lattice. We used $b=0.6$ for this comparison since we have to be in the confined phase on $4^{3}$ lattice. Figure 6 shows that the two values of $m(k)$ at a fixed $k$ are consistent with each other within errors. The same is the case for the fit parameter $\sigma b_{I}^{2}$. This is not the case for $c_{1}$ and $c_{0} b_{I}$, and this is probably due to a three parameter fit using only five data points. Sub-leading coefficients are expected to depend sensitively on the data points. Since we are primarily concerned with the value of the string tension in this paper and since all our results are based on data taken on $5^{3}$, we expect the final result to be free of finite $N$ and finite $L$ errors.

\section{Creutz ratio}

It is natural to ask how the Creutz ratio [11],

$$
\chi(K, J)=-\ln \frac{W(K, J) W(K-1, J-1)}{W(K, J-1) W(K-1, J)}
$$

performs as an observable from which to extract the string tension. If we were to use Creutz ratios, we would have smeared all links using all staples. But one can still ask how the Creutz ratio behaves 
with the asymmetrically smeared links. The $K \times K$ square Creutz ratios do not converge well as $K$ increases. It is possible the situation would be different if we had smeared all links.

Each data point in a Creutz ratio is obtained using only four different Wilson loops, i.e. four of the data points in Fig. 3. This is quite different from the analysis in this paper. Seven different Wilson loops in Fig. B are used to extract one $m(k)$ point in Fig. 2, and the loops used for different $k$ form independent sets. Then the $m(k)$ are fit to determine the string tension. Both folded and unfolded loops contribute together. This is the main reason we succeeded in extracting the string tension using the range of Wilson loops considered here. To extract the string tension using Creutz ratios, larger loops and therefore larger statistics and possibly larger $N$ would be needed.

\section{Acknowledgments}

R.N. acknowledges partial support by the NSF under grant number PHY-055375.

\section{References}

[1] R. Narayanan and H. Neuberger, Phys. Rev. Lett. 91, 081601 (2003) [arXiv:hep-lat/0303023].

[2] J. Kiskis, R. Narayanan and H. Neuberger, Phys. Lett. B 574, 65 (2003) [arXiv:hep-lat/0308033].

[3] R. Narayanan and H. Neuberger, Nucl. Phys. B 696, 107 (2004) [arXiv:hep-lat/0405025].

[4] R. Narayanan and H. Neuberger, Phys. Lett. B 616, 76 (2005) [arXiv:hep-lat/0503033].

[5] B. Bringoltz and M. Teper, Phys. Lett. B 645, 383 (2007) [arXiv:hep-th/0611286].

[6] J. Kiskis and R. Narayanan, JHEP09(2008)080 [arXiv:0807.1315].

[7] D. Karabali, C. J. Kim and V. P. Nair, Phys. Lett. B 434, 103 (1998) [arXiv:hep-th/9804132].

[8] F. Bursa and M. Teper, Phys. Rev. D 74, 125010 (2006) [arXiv:hep-th/0511081].

[9] R. Narayanan, H. Neuberger and F. Reynoso, Phys. Lett. B 651, 246 (2007) [arXiv:0704.2591 [hep-lat]].

[10] M. J. Teper, Phys. Rev. D 59, 014512 (1999) [arXiv:hep-lat/9804008].

[11] M. Creutz, Cambridge, UK: Univ. Pr. ( 1983) 169 P. ( Cambridge Monographs On Mathematical Physics) 